\title{
Research Issues in Personalization of Mobile Services
}

\author{
Muhammad Asif, John Krogstie \\ Department of Computer and Information Science, IDI \\ Norwegian University of Science and Technology, Trondheim, Norway \\ Muhammad.asif@idi.ntnu.no, krogstie@idi.ntnu.no
}

\begin{abstract}
Personalization is gaining more importance with the increase of mobile and community services. Provision of personalized mobile services can help to meet the individual needs at a time and place when and where a user needs it. Mobile services should be designed to be usable and useful to realize the benefits of personalization. It is not an easy task to satisfy the individual's goals or needs. Currently, mobile services are designed either using a client side or server- side approach. At the same time, it is raising different research issues ranging from technological to security or privacy concerns. In this work, we described the current research and development in the area of personalization of mobile services. The objective of this paper is to analyze which design approach is suitable for the personalization of mobile services. Finally, we have discussed issues and challenges related to client-side personalization vs server-side personalization and the recent trends in personalization of mobile services.
\end{abstract}

Index Terms - mobile services, personalization, user model, context model, mobile information systems

\section{INTRODUCTION}

The increasing range of mobile services raises the need for users to find out how services are beneficial. Personalization can play a significant role to select and adjust favorite services from the rapidly increasing range of mobile services. Sometimes users receive a lot of information that is irrelevant or sometimes miss urgent messages. In this setting, users demand filtered and related information according to their needs ${ }^{[1]}{ }^{[2]}$.

In general, personalization is about choice, flexibility and control, and it is about people knowing what their needs are and that people have control over how those needs are being met. For example, a personalized news service can deliver news during the day about user's working interests and entertainment news in the evening.

In another case, a user usually does shopping during the weekend and wants to receive promotions only in the weekend; a personalized advertisement service can send advertisements on the days preferred by the user. For the user, it is important to be in charge of the flow of information and services. According to ${ }^{[3]}$, delivering relevant information has two main facets: first, personalization allows users to obtain information that is adapted to their needs, goals, knowledge, interests or other characteristics. User models deliver the main parameters to select and adapt the information to the individual user. Secondly, contextualization complements personalization so that the context of use can also be taken into account. We have earlier presented a taxonomy of personalization ${ }^{[4]}$; and defined as "Personalization is a controlled process of adaptation of a service to achieve a particular goal by utilizing the user model and the context of use".

So far the focus of personalization has been on the systems or applications intended for Web or stationary computers ${ }^{[5]}$. In recent years, the focus of personalization has changed from a basic system personalization to complex service oriented personalization. Users and service providers of mobile services are facing different conceptual and technical challenges of achieving personalization. It is extremely valuable to understand what information different types of services require and what information users are willing to reveal for those services. According to ${ }^{[6]}$ there is a need to find out that for what services, what personal information the users are willing to share with the service providers in order to encompass the service provisioning based on personal information.

However, the meaning of personalization is context sensitive, and it can be beneficial to define and understand it clearly from the perspective of mobile services. As described by ${ }^{[7]}$, "the current practice of focusing on 'how to do personalization' rather than 'how can personalization be done well' suggests that the field is still in its infancy". In a way, personalization is a practice that is shaped by the designer's motives for personalization and viewpoint on "what personalization really is."

The rest of the paper is structured as follows: Section 2 describes the driving factors for personalization. The main design approaches for personalization of mobile services are described in section 3. Section 4 discusses client-side personalization and server-side 
personalization approaches and related issues. Section 5 concludes the paper.

\section{DRIVING FACTORS FOR PERSONALIZATION AND RELATED WORK}

A common characteristic of any personalization strategy is the necessity to understand and represent user needs, interests, and requirements ${ }^{[8]}$. Context-awareness and user modeling are considered as two key research areas which are contributing for adaptation and personalization of services. Both research areas have a strong role in providing personalized services and information delivery to the users. Analysis of similarities and difference between these two approaches can be useful to understand personalization better. Both research fields are contributing to personalization research particularly in personalized mobile services. A study ${ }^{[9]}$ has made similar analysis describing the relationship between these two approaches to design a context-aware Personal Digital Secretary. This study utilizes both a context model and a user model to design the application. According to the requirements of the application, they have compared both context and user model as follows:

\begin{tabular}{|l|l|l|}
\hline Issues & Context Models & User Models \\
\hline Data Acquisition & Mostly collected from all types of sensors & Mostly built from user interactions \\
\hline Coupling to Applications & Can be insulated from applications & $\begin{array}{l}\text { To be a part of an application could be } \\
\text { more efficient }\end{array}$ \\
\hline Representation & $\begin{array}{l}\text { A data model represents various context } \\
\text { elements }\end{array}$ & $\begin{array}{l}\text { A data model represents a user's facts, or } \\
\text { a behavior model or a combination of } \\
\text { both }\end{array}$ \\
\hline Time period required for data acquisition & $\begin{array}{l}\text { There is no time gap to capture a user's } \\
\text { context but may require sometimes to } \\
\text { process }\end{array}$ & $\begin{array}{l}\text { Sufficient time and interactions (Cold } \\
\text { start problem [35] required for behavior } \\
\text { model to learn a user's behavior }\end{array}$ \\
\hline
\end{tabular}

Table 1. A Comparison of Context and User Model ${ }^{[9]}$

\subsection{Context and Context-awareness}

The use of context-information can play a significant role to personalize the systems. According to Dey and Abowd ${ }^{[10]}$, "Context is any information that can be used to characterize the situation of an entity. An entity is a person, place, or object that is considered relevant to the interaction between a user and an application, including the user and the application themselves". The utilization of context is extending the problem space for the personalization of mobile services. It is required to understand which context information is necessary and how to represent it using a context model to design any context-aware application. "A system is context-aware if it uses context to provide relevant information and/or services to the user, where relevancy depends on the user's task" ${ }^{[10]}$. Context modeling is a hard problem due to its complexity and the multitude of different applications. It is required that only relevant context information should be considered for different application scenarios. Associating the context to user's preferences can play a significant role for personalized services $^{[11]}$.

Quality of the available context information is a fundamental issue. It is exceedingly hard to collect complete and accurate context information. According to ${ }^{[12]}$, it is evident that sensed context information is often inaccurate or unavailable as a result of noise or sensor failure. Moreover, user supplied information is subject to problems such as human error and staleness. Different types of context imperfections discussed by ${ }^{[12]}$ are unknown (when no information is available), ambiguous (several different values exists), imprecise (information is correct, but inexact), and erroneous (mismatch between actual and determined value). Mobile applications have the opportunity to take context into account. In ${ }^{[13]}$, a high-level categorization of context is provided as follows:

(1) The spatio-temporal context describes aspects related to time and space. It contains attributes like time, location, direction, speed and track.

(2) The environmental context captures the entities that surround the user, for example, physical objects, services, temperature, light, humidity and noise.

(3) The personal context describes the user state. It consists of the physiological and the mental contexts. The physiological context may contain information like pulse, blood pressure, and weight. The mental context may include elements such as like mood, expertise, anger and stress.

(4) The task context describes what the user is doing. The task context may be described with explicit goals or the task breakdown structure.

(5) The social context describes the social aspects of the user context, e.g., Information about friends, neighbors, co-workers, and relatives. The role that the user plays (e.g. Status and tasks to be performed) is an important aspect of social context.

(6) The information context is the information space that is available at a given time.

Table $2^{[12]}$ provides an overview of some typical properties of context information. 


\begin{tabular}{|l|l|l|l|l|}
\hline Type & Source & Persistence & Quality Issues & Source of Inaccuracy \\
\hline Sensed & $\begin{array}{l}\text { Physical and logical } \\
\text { sensors }\end{array}$ & Low & $\begin{array}{l}\text { May be inaccurate, unknown or } \\
\text { stale }\end{array}$ & $\begin{array}{l}\text { Sensor errors or failure, } \\
\text { network disconnection, delays } \\
\text { in processing }\end{array}$ \\
\hline Static & User/administrator & High & Usually none & Human error \\
\hline Profiled & Implicit or explicit & Moderate & $\begin{array}{l}\text { Prone to staleness, may be } \\
\text { unknown }\end{array}$ & Omissions to update \\
\hline Derived & Other context elements & Variable & Errors due to derivation process & $\begin{array}{l}\text { Imperfect input, depends on } \\
\text { machine learning process }\end{array}$ \\
\hline
\end{tabular}

Table 2. Properties of Context Information ${ }^{[12]}$

\subsection{User Modeling and Profiling}

Historically, user models have been used for providing adaptive and personalized services. The aim of user modeling is to capture user information such as preferences, beliefs, goals, and intentions to construct a user model ${ }^{[11]}$. User model is as an essential input for every personalization technique. The user model can either be collected by the service provider i.e.; through accumulating the information on user's preferences and interests, or imported into the system from user's personal devices.

To populate the user model, the main approaches are [14] (1) Implicit, based on the user interaction with the personalized system (2) Explicit modeling based on a questionnaire. (3) Stereotypes, which may be selected by the user or the system.

Service providers may neither cooperate, nor share the data stored in their repositories due to commercial competition ${ }^{[15]}$. Even if they agreed, there can be an issue to mediate users' models. It requires mediating mechanism that facilitates user modeling data sharing by translation and integration of the user model. Another issue that should be tackled by the mediator is integrating partial models from different domains.

User profile is a key element in personalization. Management of user profile is an essential aspect to enable personalization. Users, according to their age, concerns, and situation are likely to have different preferences and needs. The use of profiles is integral in the provision of personalized services. The need to have a well-defined user profile structure sits well with the idea that multiple applications could reuse the user profile and provide a personalized experience ${ }^{[16]}$. 3GPP Generic User Profile ${ }^{[17]}$ gives an abstract representation of a user profile which contains no data. More specific user profiles can be derived from this abstract representation of a user profile. It consists of five main components User Personal Data, User Devices, Personal User Devices, Subscribed Services and Subscribed Networks. It is further classified into User Level and Universe Level ${ }^{[18]}$ as shown in Figure 1. The User Level represents the user and the context. The Universal Level specifies formal description or schemas of all existing devices, access networks, services, and personal user devices.

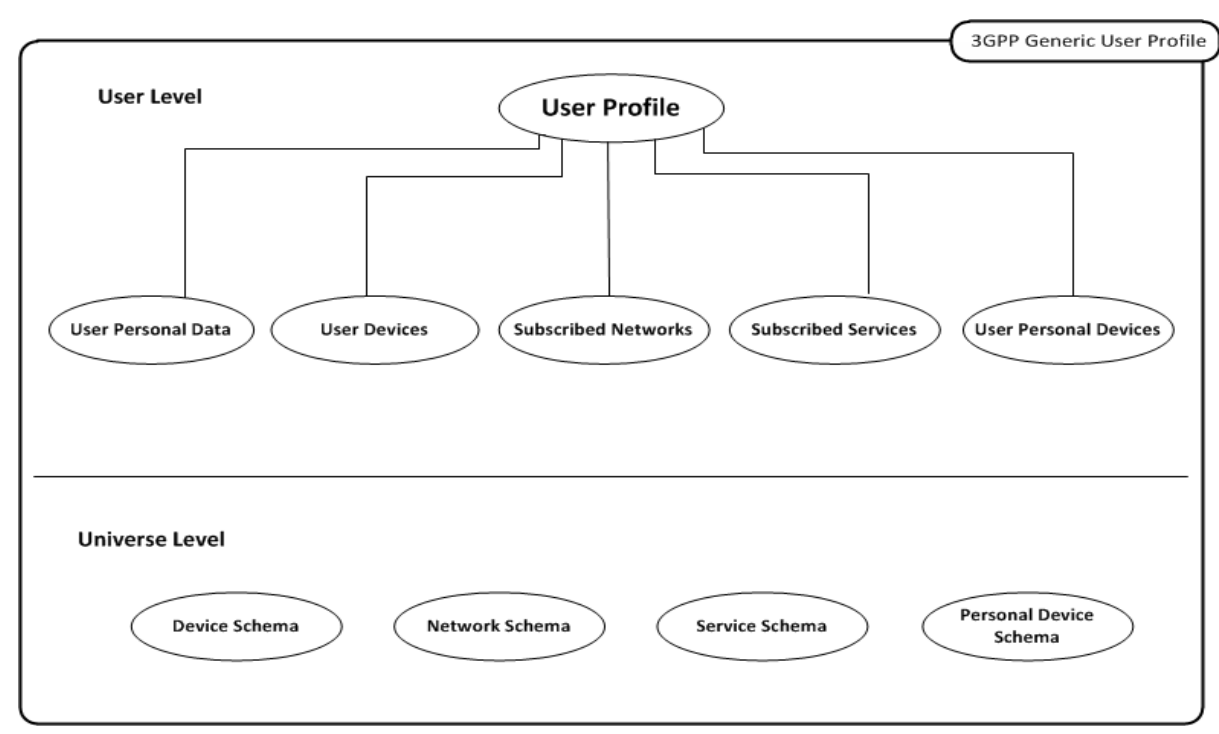

Figure 1. Generic User Profile adopted from ${ }^{[18]}$ 
In ${ }^{[19]}$ the authors have discussed different profile management and discovery issues and challenges such as profile sparseness, user profile persona, using out-ofbound data and difficulties to reuse the profile data. The user preference is personal and subjective and it needs to be handled carefully. Personal or group user models can also make it possible to represent and use information about preferences, knowledge, abilities, emotional states, and many other characteristics of a user to adapt the user experience and support ${ }^{[3]}$.

Personalization has some challenges include profile ownership, managing complexity, user acceptance and agreed standards, to name a few. There is a lack of an agreed standard for user profile capture and sharing ${ }^{[36]}$. More importantly the user profile created from one service could not be reused with another service. This might bode well with the service provider, but, from the user perspective, it is undesirable to create a user profile every time a new service requires it ${ }^{[16]}$.

\section{DESIGN APPROACHES}

Server-side personalization and client-side personalization are two distinct types of design approaches. The following sections describe these two approaches and provide a comparison of these approaches based on key elements required for personalization.

\subsection{Server-side Personalization}

The most common approach for the personalization of mobile services is performed at the server-side. For example, the study ${ }^{[9]}$ has described a system Personal Digital Secretary based on this approach. According to the authors, this approach can be useful to deal with issues like storage size and computational power in a more scalable way. It can give flexibility in adopting new contexts and modeling new behaviors.

Scalability is an important issue when capturing the available context and continuous modeling of user behavior. Machine learning approaches can also generate overhead for low computational power devices. According to ${ }^{[20]}$, it is hard for a server-based architecture to have dynamic privacy control. User modeling servers and mediations demonstrate some technological solutions but at the same time they raised privacy issues ${ }^{[21]}$. Server-based personalization requires the service providers to store and manage user model. This approach leads to the issues of scalability and optimization. Service-providers may have an incomplete or inconsistent user model which can effect personalization. It should also be noted that if the personalization is performed on the server-side implicit analysis of a user's behavior may infringe user privacy. In this way, a serverside approach must require the user's permission before gathering information this way ${ }^{[22]}$.
An advisory system ${ }^{[23]}$ uses this approach to elicit user preferences to provide personalized e-services. It has a dedicated server which keeps personalized sessions of users and generates services according to the learned behavior of users. A distributed approach is utilized in ${ }^{\text {[24] }}$ to manage the profile data of users. In the framework presented, each profile is associated with a profile manager. A user's profile can be made available to the service providers by allowing access to a respective user profile manager. This approach also applies policies and rules to handle conflicts in data. Another distributed approach ${ }^{[25]}$ is used to provide personalized news delivery on mobile devices.

\subsection{Client-side Personalization}

The increased computation power of mobile devices has opened the possibility of client-side personalization. With their increased performance and various ways of sensing their environment; it is possible to utilize it as a platform for client-side personalization. It is a most recent trend to store the user model on the mobile device to support the client-side personalization. According to ${ }^{[26]}$, it is now becoming feasible to support the client-side personalization due to the advancements in computational power of mobile devices. The authors believe that a user can have better privacy and control over data by storing the information on the mobile device. On the other hand, there can also be privacy issues through loss of devices, and applications using the personal information on the device without permission. These devices are capable of sensing aspects of the context, such as the user's location and activity. It is now possible to store a user model, communicate with the environment, and to perform client-side personalization ${ }^{[27]}$. PersonisJ ${ }^{[27]}$ is a mobile client-side user modeling framework which supports client-side personalization. A user study ${ }^{[28]}$ has shown that users prefer to store their personal information on their personal devices (smart phones) rather than store in third party servers.

Client-side solutions give a greater sense of user trust. It is easy for a user to understand that their personal information will stay under their control at all times. Trust has a positive effect on users' attitudes towards personalized mobile services. Attitude of a user towards personalized mobile services can have a positive effect on user's intention of using personalized mobile services. However, as the personalization level increases, users become more suspicious about why and how mobile service providers use their personal data, resulting in high distrust ${ }^{[29]}$. The approach usually offers the greater potential for user control over information and stronger protections ${ }^{[30]}$ 


\begin{tabular}{|c|c|c|}
\hline Issues & Server-side Personalization & Client-side Personalization \\
\hline $\begin{array}{l}\text { Scalability of Storage } \\
\text { and Processing power }\end{array}$ & $\begin{array}{l}\text { Can deal with storage and computational power } \\
\text { issues in a scalable way. Supports service based } \\
\text { approach. }\end{array}$ & $\begin{array}{l}\text { Less likely to cope with storage and } \\
\text { processing issues. This approach can partially } \\
\text { support service based approach due to } \\
\text { advancements in processing power and } \\
\text { storage capacity. }\end{array}$ \\
\hline Battery Consumption & Has no such issue & $\begin{array}{l}\text { This approach has an issue of battery } \\
\text { consumption. }\end{array}$ \\
\hline Network & $\begin{array}{l}\text { Requires continuous connectivity and secure } \\
\text { communication to connect to distributed elements. }\end{array}$ & $\begin{array}{l}\text { No need to deliver processing roles to remote } \\
\text { servers. }\end{array}$ \\
\hline Context Management & It can be easily done on dedicated context servers. & $\begin{array}{l}\text { It is hard to manage a variety of context } \\
\text { information on mobile devices. }\end{array}$ \\
\hline $\begin{array}{l}\text { Lifelong User Model } \\
\text { and Scrutability }\end{array}$ & $\begin{array}{l}\text { This approach may not be feasible for lifelong } \\
\text { scrutable user model }\end{array}$ & $\begin{array}{l}\text { This approach seems appropriate for lifelong } \\
\text { scrutable user model. This will put the user in } \\
\text { control of the personalization process. }\end{array}$ \\
\hline $\begin{array}{l}\text { Dynamic Privacy } \\
\text { Control and Security }\end{array}$ & $\begin{array}{l}\text { To deliver personal information on remote servers (or } \\
\text { third party servers) can raise privacy and security } \\
\text { issues. It is hard to achieve dynamic privacy control. }\end{array}$ & $\begin{array}{l}\text { Users can take control of personal } \\
\text { information on mobile devices anytime on the } \\
\text { go. }\end{array}$ \\
\hline $\begin{array}{l}\text { Ubiquitous User } \\
\text { Modeling }\end{array}$ & $\begin{array}{l}\text { Different parties may not agree to share user models } \\
\text { (due to commercial competition), can cause } \\
\text { replications of the user model. }\end{array}$ & $\begin{array}{l}\text { Users can take control over their profiles. } \\
\text { They can control how to share their profile } \\
\text { with service providers. Users can share a part } \\
\text { of their user model to a service provider. }\end{array}$ \\
\hline $\begin{array}{l}\text { Group } \\
\text { Personalization }\end{array}$ & $\begin{array}{l}\text { This approach can support group personalization in a } \\
\text { scalable way. User or group characteristics can be } \\
\text { explicitly or implicitly captured. }\end{array}$ & $\begin{array}{l}\text { It is difficult for this approach to handle group } \\
\text { personalization. }\end{array}$ \\
\hline $\begin{array}{l}\text { Personalized } \\
\text { Recommendations }\end{array}$ & $\begin{array}{l}\text { Server-side approach can deliver recommendations } \\
\text { based on the transactional history, and other } \\
\text { collaborative techniques. Machine learning } \\
\text { techniques and data mining techniques are usually } \\
\text { applied to build user models. }\end{array}$ & $\begin{array}{l}\text { Since the user model will primarily reside on } \\
\text { user's device, the user may not be willing to } \\
\text { share profiles for recommender systems to } \\
\text { deliver personalized recommendations. }\end{array}$ \\
\hline
\end{tabular}

Table 3. Client-side vs Server-side Personalization

Storing personal information on the server-side raises serious privacy concerns that client-side solutions do not. Client-side systems offer the primary benefit of distributed information. However, when all of the personal information is stored on the client-side, the user identity can remain anonymous to the service provider.

User model on a Key ${ }^{[21]}$ has suggested a general framework for client-side personalization by keeping the user "in control" of personal information. It has merged the user modeling server and mediation role to allow users to explicitly select the information to disclose to a particular service provider at a time. Similarly, ${ }^{[25]}$ has suggested distributed approach which stores permanent parts of user model on a server and short term user model on the user's personal device (i.e.; a mobile phone). Personalized Cultural Heritage GeoNotes ${ }^{[31]}$ is a system which also utilizes the client-side personalization approach to store user and context model of visitors on mobile devices. Hence, to create a personalized mobile service for a particular situation, it may require determining which design approach is better. However, a detailed analysis is required to understand the design requirements of a particular mobile service.

In the next section, we discussed key elements to personalization such as context management, lifelong user modeling and scrutability, ubiquity of user model, dynamic privacy control listed in table 3 and related issues. We have also discussed how these key elements should be considered while choosing a design approach such as a client-side or server-side approach. The table 3 above gives a brief comparison of both approaches and detailed discussion is presented in section 4 . 


\section{DISCUSSIONS}

Personalization has become an umbrella term and expects a clear understanding in a particular application domain such as mobile services. To achieve adequate personalization, there is a need to cope with the issues and challenges described in this paper related to clientside personalization and server-side personalization. Quality of the available context information is an important issue. It is not an easy to collect complete and accurate contextual information. Mobile services that require contextual information should be designed carefully with an understanding of the problems inherent in gathering valid context information. Imperfect context information can also create various usability issues. The problem of imperfect context information represents a significant obstacle to the success of context-aware applications and especially in personalizing the mobile services. Context modeling is a hard problem due to its complexity and the multitude of different applications.

A key challenge for service provider is to access the user model while delivering personalized services. At the same time, due to business competition and privacy issues, service providers are so far not willing to share their proprietary user modeling data. This is a big challenge for the provisioning of personalized mobile services using the server-side approach. Techniques of importing and integrating user model are another solution, but this may have other challenges in addition to privacy.

The support of lifelong user model is a key factor for lifelong personalization. Lifelong user models should be scrutable, meaning that the user can, when they want, scrutinize the user model to determine what information it holds about them. This is a foundation for enabling the user to control their model and its use, and in this way to control the personalization processes. Scrutability is as a foundation for user control over personalization. There is a need for solutions that aim for a balance between privacy and personalization. There is a variety of ways named pseudonymous personalization, scrutable personalization and dynamic personalization, they all address a handful of the main privacy concerns and achieve at least reasonably acceptable personalization [32].

Mobile device is truly a personal device and remains with user most of the time. This makes it an ideal platform for client-side personalization. The current capability of mobile device has the potential to provide unique opportunities of real-time adaptation of services in a dynamic user environment. The key change that a lifelong user model can bring is that the user can carry their user model, for example, on their mobile device. This perhaps can reduce the need for acquisition of the user model. Therefore, the user model can then be reused in other contexts. To model the user's context, especially their location and relevant aspects of their activity and attention, there can be significant technological challenges, both in collecting relevant information from sensors and then interpreting it. This may run on the user's carried device and/or an infrastructure of sensors [14]. Client-side personalization can provide a valuable foundation for lifelong user modeling, in which a user can create, edit, reuse, and extend their user model throughout their digital life experiences ${ }^{[27]}$. It seems that having an integrated, standard, personal "lifelong" user model can provide a starting point for personalization in several forms. The user model can be stored physically or logically on the user's mobile device, perhaps with parts made available to different domains. However, it is essential to be able to use user modeling data across domains ${ }^{[14]}$.

The personalization research area is somehow fragmented ${ }^{[5]}$. We can clearly see that there is a requirement for a conceptual personalization framework in the context of mobile personalization. According to [33], this is an increasing trend in the use of personalization technology. It is analogous to speeding up a train whose direction is unknown. Moreover, the author has stated that the first and foremost challenge for the personalization research community is reaching a consensus of a common frame of reference for personalization.

The shift of research focus from technology oriented towards the user-centered approach raises the issue of individual privacy and data protection ${ }^{[21]}$. The information required to personalize the services has raised the concerns for "right to privacy". Mobile users are facing a dilemma: while they demand more customized services, they are increasingly concerned about privacy infringements and how their information is being used by mobile service providers. Therefore, mobile users are more suspicious of new personalized services ${ }^{[29]}$. Acquisition, processing, and storage of personal data ubiquitously may require an intensified consideration of user demands to security, privacy, and anonymity ${ }^{[34]}$. According to ${ }^{[6]}$, there is a need to find out what services, what personal information the users are willing to share with the surrounding services in order to encompass the service provisioning based on personal information. A tradeoff may require exploiting how much personal information a user is willing to share in order to continue using a service. However, it is difficult to achieve balance and guarantee of this tradeoff. One possible solution is to shift the control to the users over their personal information and make explicit the tradeoff between benefits and risks according to level of involvement. In this case, client-side personalization seems a better option for the personalization of mobile services.

\section{CONCLUSIONS}

Context-awareness and user modeling are enabling a number of services to support personalization. Both research areas have various applications in different domains. Personalization is another dimension of services enabled by these research areas. In addition to getting the benefits of these disciplines, personalization 
of mobile services has also inherited the challenges. The challenges of these areas extend the problem space for personalized mobile services. However, there is a need to reduce these challenges and develop the solution possibilities by achieving tradeoff between the design choices. This paper aims at summarizing different approaches, solutions, issues and challenges so far for personalized mobile services. Summarizing the study and state of art of personalization in mobile services can lead to a number of high-level considerations for the next generation of personalized mobile services. This article is an attempt to investigate and compare client-side personalization and server-side personalization approaches to provide a way to the next generation of personalized mobile services.

\section{REFERENCES}

[1] Fausto, M. and P. Alberto. Context planning and user profiling in mobile services. 2010. IEEE.

[2] Kim, W., Personalization: Definition, status, and challenges ahead. Journal of object technology, 2002. 1(1): p. 29-40.

[3] Zimmermann, A., M. Specht, and A. Lorenz, Personalization and context management. User Modeling and User-Adapted Interaction, 2005. 15(3): p. 275-302.

[4] Asif, M. and J. Krogstie, Taxonomy of Personalization in Mobile Services, in Proceedings of the 10th IADIS International Conference e-Society 2012, ISBN: 978-972-8939-67-02012: Berlin, Germany.

[5] Sunikka, A. and J. Bragge. What, Who and Where: Insights into Personalization. in Hawaii International Conference on System Sciences, Proceedings of the 41st Annual. 2008.

[6] Heikkinen, K., et al., Personalized View of personal information. WSEAS Transactions on Information Science and Applications, 2004. 2(4).

[7] Fan, H. and M.S. Poole, What is personalization? Perspectives on the design and implementation of personalization in information systems. Journal of Organizational Computing and Electronic Commerce, 2006. 16(3-4): p. 179-202.

[8] Perugini, S. and M.A. Gonçalves, Recommendation and personalization: a survey. Journal of Intelligent Information Systems, 2002.

[9] Byun, H.E. and K. Cheverst. Exploiting user models and context-awareness to support personal daily activities. 2001.

[10] Abowd, G, et al. Towards a better understanding of context and context-awareness. 1999. Springer.

[11] Coutand, O., A Framework for Contextual Personalised Applications, 2009, kassel university press GmbH: Germany. p. 240.

[12] Henricksen, K. and J. Indulska. Modelling and using imperfect context information. 2004. Ieee.

[13] Krogstie, J., et al., Usable M-Commerce Systems: The Need of Model-Based Approaches. Advances in mobile commerce technologies, 2003: p. 190.

[14] Kuflik, T., J. Kay, and B. Kummerfeld, Lifelong Personalized Museum Experiences. Proc. Pervasive User Modeling and Personalization (PUMP'10), 2010.

[15] Berkovsky, S., T. Kuflik, and F. Ricci, Mediation of user models for enhanced personalization in recommender systems. User Modeling and UserAdapted Interaction, 2008. 18(3): p. 245-286.

[16] Bilchev, G., et al., Personal spaces - ePerSpace. BT technology journal, 2005. 23(3): p. 226-238.

[17] 3GPP, 3rd Generation Partnership Project. , in Data Description Method (DDM) - 3GPP Generic User Profile (GUP). Technical specification of Technical Specification Group Terminals, Version 10.0.0 2011.

[18] Bartolomeo, G., et al. Handling User Profiles for the Secure and Convenient Configuration and Management of Mobile Terminals and Services. 2005. IEEE.

[19] Ghosh, R. and M. Dekhil. Discovering user profiles. in Proceedings of the 18th international conference on World wide web. 2009. Madrid, Spain: ACM.

[20] Juong-Sik, L. and U. Chandra. Mobile phone-tophone personal context sharing. in Communications and Information Technology, 2009. ISCIT 2009. 9th International Symposium 2009.

[21] Kuflik, T. and K. Poteriaykina, User model on a key, Proceedings of the 20th ACM conference on Hypertext and hypermedia2009, ACM: Torino, Italy. p. 371-372.

[22] Lee, W.P., Deploying personalized mobile services in an agent-based environment. Expert Systems with Applications, 2007. 32(4): p. 1194-1207.

[23] Jannach, D. and G. Kreutler. Personalized user preference elicitation for e-services. 2005. IEEE.

[24] Agostini, A., et al., Towards highly adaptive services for mobile computing. Mobile information systems, 2005: p. 121-134.

[25] Papadogiorgaki, M., et al. Distributed user modeling for personalized news delivery in mobile devices. 2007. IEEE.

[26] Wasinger, R., et al., Client-side User Modelling across Multiple Mobile Applications. 2011.

[27] Gerber, S., et al., PersonisJ: Mobile, Client-Side User Modelling, Adaptation, and Personalization, Springer Berlin / Heidelberg. p. 111-122, 2010.

[28] Juong-Sik, L., et al. Personal Relationship Management via Mobile Phone. in Consumer Communications and Networking Conference (CCNC), 2010 7th IEEE. 2010.

[29] Shuk Ying, H. and S.B. Bull. Users' Adoption of Mobile Services: Preference and Location Personalization. in 2010 5th International Conference on Computer Sciences and Convergence Information Technology (ICCIT 2010), 30 Nov.-2 Dec. 2010. Piscataway, NJ, USA: IEEE.

[30] Mulligan, D. and A. Schwartz. Your place or mine?: privacy concerns and solutions for server and client- 
side storage of personal information. 2000. ACM.

[31] Brem, D., et al. Personalized Cultural Heritage GeoNotes. 2010.

[32] Wang, Y. and A. Kobsa, Technical solutions for Privacy-Enhanced personalization. Intelligent User Interfaces: Adaptation and Personalization Systems and Technologies, 2009: p. 326-353.

[33] Blom, J., Challenges for User-Centric Personalization Research. Designing Personalized User Experiences in eCommerce, 2004, Springer Netherlands. p. 333-348.

[34] Heckmann, D., Ubiquitous user modeling for situated interaction. User Modeling 2001: p. 280282.

[35] Adomavicius, G. and A. Tuzhilin, Personalization technologies: a process-oriented perspective. Communications of the ACM, 2005. 48(10): p. 8390.

[36] Hella L, Krogstie J (2010) A Structured Evaluation to Assess the Reusability of Models of User Profiles. Paper presented at the EMMSAD - Conference on Evaluating Modeling Methods in Systems Analysis and Design, Hammamet, Tunis, 7-8/6.

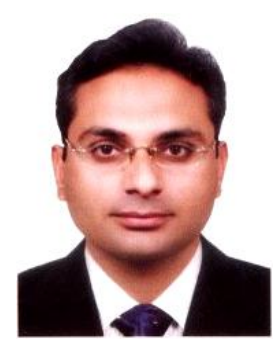

Muhammad Asif is a $\mathrm{PhD}$ student in the Department of Computer and Information Science (IDI), Norwegian University of Science and Technology (NTNU), Norway. He completed his master degrees in information system engineering in 2010 at Norwegian University of Science and Technology. His research interests include mobile information systems, mobile computing, user modeling, and model driven information systems engineering.

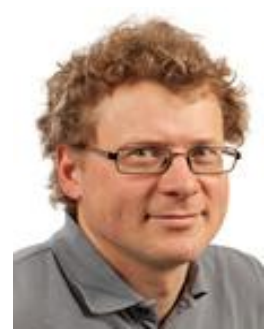

John Krogstie holds a $\mathrm{PhD}$ (1995) and an MSc (1991) in information systems from the Norwegian University of Science and Technology (NTNU), where he is currently a full professor in information systems. John Krogstie is the Norwegian representative for IFIP TC8 and Chair of IFIP WG 8.1 on information system design and evaluations. He has published around 150 refereed papers in journals, books and archival proceedings since 1991. 